\title{
Development of Molecularly Imprinted Olanzapine Nano-particles: In Vitro Characterization and In Vivo Evaluation
}

\author{
Nersi Jafary Omid, ${ }^{1}$ Hoda Morovati, ${ }^{1}$ Mohsen Amini, ${ }^{2}$ Ahmad-Reza Dehpour, ${ }^{3}$ Alireza Partoazar, ${ }^{3}$ \\ Morteza Rafiee-Tehrani, ${ }^{1}$ and Farid Dorkoosh ${ }^{1,4,5}$
}

Received 27 September 2015; accepted 9 January 2016; published online 2 February 2016

\begin{abstract}
Molecularly imprinted nano-particles (MINPs) selective for olanzapine were prepared using methacrylic acid (MA) as monomer, ethylene glycol dimethacrylate (EGDMA) as a cross-linker, and 2,2azobis (2-isobutyronitrile) (AIBN) as the initiator in 36 different ratios. The reaction runs with considerable fine powder formation were selected for further binding and selectivity studies. The MINP with the best selectivity (MINP-32) was chosen for further structural characterization by Fourier transform infrared spectroscopy (FT-IR), thermal gravimetric analysis (TGA), scanning electron microscopy (SEM), adsorption-desorption isotherm for specific surface area, volume and average pore diameter determination. All characterization methods confirmed the successful formation of MINP. The optimum conditions for maximum template loading on the MINP-32 were found by experimental design using response surface methodology (RSM) and choosing absorbent amount, $\mathrm{pH}$, and time as the main factors. MINPs with maximum template loading also indicated significant selectivity between template and its analog (clozapine). The release profile demonstrated a maximum release of about $95 \%$ after $288 \mathrm{~h}$ for MINP-32 in comparison with about $94 \%$ after $120 \mathrm{~h}$ for non-MINP-32. The same slow release of drug from MINP32 was also observed during animal study of the plasma level of template, $20-28 \mu \mathrm{g} / \mathrm{ml}$ versus $5-10 \mu \mathrm{g} / \mathrm{ml}$. The MINP-32 of this study represents a desirable ability to keep the memory of the template with significant selectivity and good capability to control the release of template in vitro and in vivo and hence could be a promising drug delivery system.
\end{abstract}

KEYWORDS: experimental design; molecularly imprinted polymers; nano-particles; olanzapine; schizophrenia.

\section{INTRODUCTION}

Synthetic polymers are one of the most important materials with diverse properties and application in many aspects of human daily life. Due to technological improvement and thanks to merging in pharmaceutical sciences during the recent 30 years, polymers have found a wide range of applications in this field with functions that encompass simple tasks such as solubilizer, stabilizer, tablet film coater and binder to more complicated ones such as drug release modulation, drug targeting and responsiveness to environmental stimuli as smart polymers (1-3). Polymers

\footnotetext{
$\overline{{ }^{1} \text { Department of Pharmaceutics, Faculty of Pharmacy, Tehran Univer- }}$ sity of Medical Sciences, Tehran, Iran.

${ }^{2}$ Department of Medicinal Chemistry, Faculty of Pharmacy, Tehran University of Medical Sciences, Tehran, Iran.

${ }^{3}$ Department of Pharmacology, Faculty of Medicine, Tehran University of Medical Sciences, Tehran, Iran.

${ }^{4}$ Pharmaceutical Products Technology Units Incubator, No. 1462, Kargar Ave., Tehran, Iran1439804448.

${ }^{5}$ To whom correspondence should be addressed. (e-mail: dorkoosh@tums.ac.ir)
}

in drug delivery perform their function either by governing time control or location control (4).

Molecularly imprinted polymers (MIPs), among other polymers, have been considered in pharmaceutical technology and became attractive due to their unique characteristics. They are polymeric networks which have memorized the configuration of a host molecule known as template. Molecular imprinting is not a new know-how and its technology originated 70-80 years ago but has changed and improved significantly during this period (5). At the beginning, these polymers were applied in different chemical purification and analytical separation techniques and processes (6-8), as scavenger for undesired compounds in food industries (9), as a tool for drug discovery (10), and as enzyme-like catalysts (11). Recently, MIPs are considered as a drug delivery tool as not only they can have better control on drug release in comparison to conventional polymers due to their selective affinity to the host molecule but also they can achieve responsiveness to environmental conditions by employing responsive comonomers in their structure and hence regulate the drug release from the polymeric network (12). MIPs can even be applied in conventional dosage forms such as tablets to modulate the drug release in response to a biomarker (13). 
One of the main features of MIPs is the ease of synthesis. Generally, a template is mixed with one or more functional monomers to make a primary complex which will then be cross-linked by a suitable cross-linker. Different strategies are used to prepare MIPs including covalent, semi-covalent, non-covalent, metal ions mediated, and nonpolar interaction (14). Each of these methods has its own advantages and disadvantages. In covalent method, less non-specific recognition sites are formed and a wider range of polymerization conditions is tolerable; however, a chemical modification should be done on template before and after MIP preparation. Non-covalent strategy is attractive because of its simplicity and its potential to cover a wide range of chemical functionalities. Moreover, non-covalent method form more stable cavity to maintain conformational structure in the absence of the template and impose more flexibility to the structure of polymer for ease of template realization and establishing fast equilibrium between the release and re-uptake of the template (15). However, this method has its own drawbacks such as heterogeneity of recognition sites, more number of nonspecific binding sites and low yield of receptor sites with high affinity.

Olanzapine, as an example of new generation antipsychotics, has been considered as first line therapeutic for treatment of schizophrenia. Schizophrenia is a mental disorder in which the patient cannot think properly and has poor response to emotions. It is characterized by auditory hallucinations, paranoid or bizarre delusions, or disorganized speech and thinking, and it is accompanied by significant social or occupational dysfunction (16). Both genetic and environmental factors, such as premature birth, low birth weight, and prenatal hypoxia, social isolation, migrant status, and urban life, are important in development of symptoms of the disorder (17). Dopamine D2 receptor antagonists have been used to treat this disorder for years as first generation drugs. Due to the side effects of these drugs, the second generation drugs such as olanzapine has been developed in the recent decade.

Usually, elderly patients who suffer from schizophrenia and use olanzapine as long-term therapy may also have other diseases and disorders and should use several medications at the same time. The lesser the number of times a patient has to take different medicines per day the better he or she will follow the treatment with more compliance (18). Therefore, controlled release formulations are considered in this regard. Different techniques have been employed for controlling the drug release from a formulation which can govern the release process by various types of mechanisms such as diffusion, dissolution, osmosis, swelling, chemical interactions, hydrodynamic pressure, $\mathrm{pH}$, and so on (19). Molecular imprinting is a type of chemical interaction which has recently been considered as a tool for drug delivery.

In this research work, an MIP system was synthesized and characterized employing non-covalent strategy using olanzapine as template, methacrylic acid (MA) as monomer, and ethylene glycol dimethacrylate (EGDMA) as a crosslinker in different solvent systems as porogen to study the morphology, loading efficacy, selectivity, in vitro release and in vivo controlled release efficacy of drug molecule.

\section{MATERIALS AND METHODS}

\section{Materials}

Olanzapine and clozapine were received as a gift from Aboureyhan Pharmaceutical Company, Tehran-Iran. Methacrylic acid (MA), ethylene glycol dimethacrylate (EGDMA), 2,2-azobis (2-isobutyronitrile) (AIBN), acetonitrile, methanol, ethanol, acetone, hydrochloric acid, chloroform, glacial acetic acid, ethyl acetate, and ascorbic acid were purchased from Merck, Germany. Dialysis bag with molecular weight cut off (MWCO) 12,000 g/mol, heparin sodium, ketamine hydrochloride, and xylazine hydrochloride were purchased from Sigma-Aldrich, USA. MA was further purified by distillation while other reagents were used as received. Male Wistar rats $(210 \pm 20 \mathrm{~g})$ were used for animal studies.

\section{Methods}

\section{Preparation of MIP Nano-particles (MINPs)}

Precipitation polymerization was used to synthesize MINPs. Through a preliminary screening, specified amounts of template, monomer and cross-linker (Table I) were placed in a 100-ml polymerization tube and dissolved in a $35 \mathrm{ml}$ mixture of chloroform and acetonitrile as solvent system and mixed overnight. The tube was then purged with nitrogen to replace oxygen, $0.02 \%$ total weight of monomer and crosslinker of initiator was added, sealed, and kept for $22 \mathrm{~h}$ in a water bath at a temperature of $60^{\circ} \mathrm{C}$ with continuous stirring. At the end of the polymerization, the solvent system was evaporated under vacuum and the product was washed several times with a mixture of methanol to acetic acid $(9: 1)$ for $1 \mathrm{~h}$ until no template remained within the polymer structure (the UV absorption of the supernatant at $252 \mathrm{~nm}$ was no longer observed). The resulting powder was then washed twice by purified water and once with acetone and was left to dry at room temperature. Similar steps were performed without using template to obtain non-MINPs.

\section{Characterization of MINPs}

Ultraviolet Spectroscopy. Drug concentration measurements for control of effective template removal after washing polymer system, loading experiments and selectivity were performed by ultraviolet spectroscopy (UV) spectroscopy using a UV/VIS spectrophotometer at 252 and $220 \mathrm{~nm}$ for olanzapine and clozapine, respectively (Optizem 2120 UV PLUS, Korea).

Fourier Transform Infra Red Spectroscopy (FT-IR). FTIR spectrum of MINP-32 and non-MINP-32 was obtained in solid state ( $\mathrm{KBr}$ as filler), at room temperature in the range 
Table I. Mole Ratio of Template: Monomer: Cross-Linker, and Mixture of System Solvents Used to Prepare the MIP Nano-particles

\begin{tabular}{|c|c|c|c|c|c|c|}
\hline Run numbers & Template (mmol) & MA (mmol) & EGDM $(\mathrm{mmol})$ & $\mathrm{CHCl}_{3}(\%)$ & $\operatorname{AcCN}(\%)$ & Reaction product \\
\hline 1 & 1 & 2 & 16 & 100 & 0 & Rigid mass \\
\hline 2 & 1 & 2 & 16 & 85 & 15 & Low level of fine particles \\
\hline 3 & 1 & 2 & 16 & 50 & 50 & Low level of fine particles \\
\hline 4 & 1 & 2 & 32 & 100 & 0 & Rigid mass \\
\hline 5 & 1 & 2 & 32 & 85 & 15 & Low level of fine particles \\
\hline 6 & 1 & 2 & 32 & 50 & 50 & Low level of fine particles \\
\hline 7 & 1 & 2 & 64 & 100 & 0 & Rigid mass \\
\hline 8 & 1 & 2 & 64 & 85 & 15 & Low level of fine particles \\
\hline 9 & 1 & 2 & 64 & 50 & 50 & Low level of fine particles \\
\hline 10 & 1 & 4 & 16 & 100 & 0 & Rigid mass \\
\hline 11 & 1 & 4 & 16 & 85 & 15 & High level of fine particles \\
\hline 12 & 1 & 4 & 16 & 50 & 50 & Low level of fine particles \\
\hline 13 & 1 & 4 & 32 & 100 & 0 & Rigid mass \\
\hline 14 & 1 & 4 & 32 & 85 & 15 & High level of fine particles \\
\hline 15 & 1 & 4 & 32 & 50 & 50 & Low level of fine particles \\
\hline 16 & 1 & 4 & 64 & 100 & 0 & Rigid mass \\
\hline 17 & 1 & 4 & 64 & 85 & 15 & High level of fine particles \\
\hline 18 & 1 & 4 & 64 & 50 & 50 & Low level of fine particles \\
\hline 19 & 1 & 6 & 16 & 100 & 0 & Rigid mass \\
\hline 20 & 1 & 6 & 16 & 85 & 15 & High level of fine particles \\
\hline 21 & 1 & 6 & 16 & 50 & 50 & Low level of fine particles \\
\hline 22 & 1 & 6 & 32 & 100 & 0 & Rigid mass \\
\hline 23 & 1 & 6 & 32 & 85 & 15 & High level of fine particles \\
\hline 24 & 1 & 6 & 32 & 50 & 50 & Low level of fine particles \\
\hline 25 & 1 & 6 & 64 & 100 & 0 & Rigid mass \\
\hline 26 & 1 & 6 & 64 & 85 & 15 & High level of fine particles \\
\hline 27 & 1 & 6 & 64 & 50 & 50 & Low level of fine particles \\
\hline 28 & 1 & 8 & 16 & 100 & 0 & Rigid mass \\
\hline 29 & 1 & 8 & 16 & 85 & 15 & High level of fine particles \\
\hline 30 & 1 & 8 & 16 & 50 & 50 & Low level of fine particles \\
\hline 31 & 1 & 8 & 32 & 100 & 0 & Rigid mass \\
\hline 32 & 1 & 8 & 32 & 85 & 15 & High level of fine particles \\
\hline 33 & 1 & 8 & 32 & 50 & 50 & Low level of fine particles \\
\hline 34 & 1 & 8 & 64 & 100 & 0 & Rigid mass \\
\hline 35 & 1 & 8 & 64 & 85 & 15 & High level of fine particles \\
\hline 36 & 1 & 8 & 64 & 50 & 50 & Low level of fine particles \\
\hline
\end{tabular}

4000-400 $\mathrm{cm}^{-1}$ using Nicolet FT-IR Magna 550 spectrophotometer (Thermo Electron Corporation, USA).

Thermal Gravimetric Analysis. Thermal gravimetric analysis (TGA) was used to assess the effect of structural configuration due to memorization of template inside the polymer backbone and justification of molecular imprinting employing Perkin Elmer Pyris Diamond TG/DTA analyzer (PerkinElmer Life and Analytical Sciences, USA). The temperature range was set from 25 to $700^{\circ} \mathrm{C}$ with increments of $20^{\circ} \mathrm{C}$ per minute under $\mathrm{N} 2$ gas.

Size and Zeta Potential. The size and poly-dispersity of MINP-32 was measured by photon correlation spectroscopy using a Zetasizer 3000HS (Malvern Instruments, Malvern, United Kingdom). The sample was diluted by filtered purified water and scattering angle was set at $90^{\circ}$ for measurement. The measurement was done three times at $25^{\circ} \mathrm{C}$ and the mean values $\pm \mathrm{SD}$ were reported.

The charge of the MINP-32 was measured by laser Doppler anemometry using a Zetasizer 3000HS (Malvern Instruments, Malvern, United Kingdom). The sample was diluted by filtered purified water which then placed in the electrophoretic cell, where a potential of $150 \mathrm{mV}$ was established. The mean of at least 3 runs was used to be reported.

Scanning Electron Microscopy. The morphology of the MINP-32 and non-MINP-32 were assessed by scanning electron microscopy (SEM) (Philips XL30 scanning microscope, Philips, the Netherlands). An aqueous suspension of the particles was fixed, dried, and coated with gold. This operation in DC-magnetron sputtering was carried out for $10 \mathrm{~min}$ in DC plasma condition using Argon gas with DC voltage of $6 \mathrm{kV}$ and DC current of $6 \mathrm{~mA}$. The accelerator voltage for scanning was $20 \mathrm{kV}($ Vacc $=20 \mathrm{kV})$. Diameter of particles was determined using CLEMEX® particle image analysis software package.

Adsorption-Desorption Isotherm. Porosity and surface morphology of the MINP-32 were studied by measuring liquid $\mathrm{N} 2$ adsorption-desorption isotherm in a microporosimeter (BELSORP Mini, Japan). One hundred fifty milligrams of sample was dried in vacuo at $100^{\circ} \mathrm{C}$ for $3 \mathrm{~h}$ before analysis. Analysis was performed at $77 \mathrm{~K}\left(-196^{\circ} \mathrm{C}\right)$. The specific surface 
area was measured using the Brunauer-Emmett-Teller (BET) methodology. The specific pore volume and average pore diameter were calculated employing Barrett, Joyner, and Halenda (BJH) method.

\section{Loading Experiments}

Preliminary loading experiments were done as follows: $30 \mathrm{mg}$ of washed MINPs and non- MIPNs were dispersed in $8 \mathrm{ml}$ of a solution containing $250 \mu \mathrm{g} / \mathrm{ml}$ olanzapine in phosphate buffer to acetonitrile $4: 1(v / v, \mathrm{pH}=5.0)$ by sonication for $6 \mathrm{~min}$ and then stirred gently for $60 \mathrm{~min}$. The liquid phase was then separated by centrifugation at $5000 \mathrm{rpm}$ for $10 \mathrm{~min}$ and analyzed by UV at $252 \mathrm{~nm}$ (validated for quantitative analysis - data is not shown). The amount of drug bound to the polymers was calculated using the following equation:

$D b(\%)=\frac{\mathrm{CA}-\mathrm{CB}}{\mathrm{CB}} \times 100$

Where $D b$ is the drug bound (loaded) to the polymers, $\mathrm{CA}$ is the initial drug concentration, and $\mathrm{CB}$ is the concentration of the drug in the liquid phase.

The MINP with maximum capacity of drug loading (MINPs-32, please refer to "RESULTS" and "DISCUSSION" and Fig. 2) was chosen and the optimum conditions for the loading were found by response surface methodology (RSM) using Box-Behnken methodology employing Design Expert 6.0.10 software (Stat-Ease, Inc., Minneapolis, USA). The effects of polymer (absorbent) amount, $\mathrm{pH}$ and time as the main factors were studied on drug loading as response. The lower and upper levels of the factors are chosen based on the results of preliminary studies which are as follows: 10 and $100 \mathrm{mg}$ for polymer amount, 1 and $11 \mathrm{for} \mathrm{pH}$, and 30

Table II. Experimental Matrix Design for Binding Study with Results

\begin{tabular}{lllll}
\hline Run & $X_{i}$ & $X_{i i}$ & $X_{i i i}$ & $Y$ \\
\hline 1 & 55 & 11 & 120 & 89.0 \\
2 & 55 & 11 & 30 & 58.0 \\
3 & 10 & 11 & 75 & 39.0 \\
4 & 55 & 6 & 75 & 31.0 \\
5 & 10 & 1 & 75 & 2.0 \\
6 & 55 & 1 & 30 & 0.5 \\
7 & 55 & 6 & 75 & 29.0 \\
8 & 100 & 6 & 30 & 35.0 \\
9 & 10 & 6 & 30 & 16.0 \\
10 & 55 & 1 & 120 & 40.0 \\
11 & 10 & 6 & 120 & 13.0 \\
12 & 55 & 6 & 75 & 19.0 \\
13 & 55 & 6 & 75 & 33.0 \\
14 & 100 & 11 & 75 & 98.0 \\
15 & 55 & 6 & 75 & 30.0 \\
16 & 100 & 6 & 120 & 47.0 \\
17 & 100 & 1 & 75 & 15.0 \\
\hline
\end{tabular}

$X_{i}$ polymer amount $(\mathrm{mg}), X_{i i} \mathrm{pH}, X_{i i i}$ time (min), $Y$ drug loading and $120 \mathrm{~min}$ for time. Experiment matrix design and responses are presented in Table II. Experimental runs were randomized to minimize the errors due to uncontrolled factors. Analysis of variance (ANOVA) was applied to investigate model fitness and effects of individual factors and their interactions on response. Based on RSM results, selectivity of MINP-32 was tested in comparison with its counterpart non-MINP by dispersing $79 \mathrm{mg}$ of washed MINP-32 and non-MINP-32 in $30 \mathrm{ml}$ solution containing $250 \mu \mathrm{g} / \mathrm{ml}$ olanzapine or clozapine at $\mathrm{pH}=10.90$, and stirred for $79 \mathrm{~min}$ at room temperature. The liquid phase was separated by centrifugation at $5000 \mathrm{rpm}$ for $10 \mathrm{~min}$ and analyzed for free olanzapine and clozapine by UV spectroscopy at 252 and $220 \mathrm{~nm}$, respectively (validated for quantitative analysis, data are not shown). The amount of loaded drug was calculated by the aforementioned formula.

\section{In Vitro Study}

Release Profile. Olanzapine was loaded on washed MINP-32 and non-MINP-32 as mentioned above. The precipitate after centrifugation was freeze dried and dispersed in $4 \mathrm{ml}$ phosphate buffer saline (containing $\mathrm{NaCl}=8 \mathrm{~g}$, $\mathrm{KCl}=0.2 \mathrm{~g}, \mathrm{Na} 2 \mathrm{HPO} 4=1.44 \mathrm{~g}, \mathrm{KH} 2 \mathrm{PO} 4=0.25 \mathrm{~g}$ in 1 , adjusted at $\mathrm{pH}=7.4)$ in a dialysis bag and floated in $16 \mathrm{ml}$ of the same medium and shook at $37^{\circ} \mathrm{C} \pm 0.5$. Onemilliliter samples were withdrawn at $0,1,2,4,8,12,24 \mathrm{~h}$ and, afterwards, once a day for 14 days with medium substitution to keep the sink condition. Drug release was determined by HPLC using KH2PO4 $0.75 \mathrm{mMol}$, acetonitrile and methanol in a ratio of 55:40:5 adjusted at $\mathrm{pH}=4.0$ with orthophosphoric acid as mobile phase with a flow rate of $0.8 \mathrm{ml} / \mathrm{min}$, a $250 \mathrm{~mm} \times 4.6 \mathrm{~mm}, 10 \mu \mathrm{m} \mathrm{C} 8$ column [Lichrosorb RP-8 (10)], and UV detection at $227 \mathrm{~nm}$. Injection volume was $20 \mu l$.

Release Mechanism. The mechanism of drug release from MINP-32 was evaluated using common mathematical models. Among them, models with better fitting results (regression coefficients, $\left.R_{\mathrm{sqr}}>0.95\right)$ were chosen for further analysis.

Higuchi model explains drug release from a matrix system mathematically which was first used for planar systems and then developed to different geometrics and porous systems. Drug release by diffusion according to Fick's law from a number of modified release pharmaceutical dosage forms is well described by this model (20). It relates the drug release to square root of time and is presented by the following equation (21):

$\frac{M t}{M \infty}=K_{H} t^{1 / 2}$

in which $M t / M \infty$ is the fractional drug release, $K_{H}$ is the Higuchi constant and $t$ is the time.

Korsmeyer and Peppas mathematical model simply relates time and release exponentially to each other to describe drug release from a polymeric system (22). This model has a strong ability to differentiate between different release mechanisms such as Fickian diffusion, Non-Fickian transport, case II (relaxation or swelling controlled) 
transport, and supper case II (erosion controlled) transport. The model is presented as follows:

$\frac{M t}{M \infty}=K_{p} t^{n}$

in which $M t / M \infty$ is the fractional drug release, $K_{p}$ is the model constant, $t$ is the time, and $n$ is the release exponent which its value characterizes the release mechanism.

Baker and Lonsdale mathematical model has been developed from the Higuchi model to explain the drug release from spherical matrices such as microcapsules or microspheres (20) according to the equation:

$\frac{3}{2}\left[1-\left(1-\frac{M t}{M \infty}\right)^{\frac{2}{3}}\right] \frac{M t}{M \infty}=k 1$

in which $M t / M \infty$ is the fractional drug release and $k 1$ is the release rate constant.

Thermal Stability Study of the MINP-32. A suitable amount (about $450 \mathrm{mg}$ ) of MINP-32 was placed in a small beaker and kept at $50 \pm 2^{\circ} \mathrm{C} / 90 \pm 5$ relative humidity $(\mathrm{RH})$ for one month. The assay and impurity profile of olanzapine was measured at the beginning, after 15 and 30 days of the study by HPLC method as described under "2.2.4.1 Release profile."

\section{In Vivo Study of MINPs in Rat}

All animal studies were performed in compliance with "The Basis of Laboratory Animal Science" prepared and published by Royan institute, 2013, ISBN: 978-600-92587-8-9. Twelve male Wistar rats (average body weight $210 \pm 20 \mathrm{~g}$ ) were divided in two groups of 6 . A dispersion of MINP-32 containing $5 \mathrm{mg} / \mathrm{Kg}$ olanzapine in water for injection (after sonication for $24 \mathrm{~h}$ ) was injected intramuscularly (IM) to the left leg of the animals in one group (test) and $5 \mathrm{mg} / \mathrm{Kg}$ olanzapine alone was injected in the same way to the other group (control). The animals were anesthetized using ketamine (50 mg/kg body weight) and xylazine $(5 \mathrm{mg} / \mathrm{kg}$ body weight). After the onset of anesthesia (about $10 \mathrm{~min}$ ) $1 \mathrm{ml}$ blood samples were withdrawn directly from the heart of the fully anesthetized animals within $30 \mathrm{~min}$ at the beginning and then 24, 96, 168 and $240 \mathrm{~h}$ after injection.

Drug extraction was performed immediately after each sampling as follows: blood samples were transferred to heparinized tubes and centrifuged at $4000 \mathrm{rpm}$ for $20 \mathrm{~min}$. The supernatant liquid was transferred to another micro-tube and $1 \mathrm{ml}$ ethyl acetate was added and vortexed for $1 \mathrm{~min}$ and centrifuged at $5000 \mathrm{rpm}$ for $20 \mathrm{~min}$. The upper liquid evaporated at $60^{\circ} \mathrm{C}$ under vacuum and stored at $-80^{\circ} \mathrm{C}$ until analysis. Analysis was performed by HPLC with the same method aforementioned by dissolving the samples in $1 \mathrm{ml}$ of mobile phase.
Statistical Analysis

All data is presented as mean \pm standard deviation from three independent experiments. Statistical significant differences were assessed employing SPSS 11.5 software, using the Student $t$ test with probability values $<0.05$ assumed significant.

\section{RESULTS}

\section{MINPs Formation and Characterization}

The synthesis of the MINPs is schematically illustrated in Fig. 1. Non-covalent strategy was selected due to its benefits as aforementioned in the introduction. Different ratios of monomer and cross-linker against a fix amount of template as well as different solvents alone or in a mixture were examined to prepare the MINPs as presented in Table I. The preliminary criterion of selection was formation of MINPs as a fine powder directly in reaction mixture. Reaction in acetonitrile alone did not result in any polymer while a rigid mass resulted in chloroform alone. Therefore, only the product of reaction runs resulted in high level of fine particles were selected for binding experiments. The loading efficacy of the selected MINPs and counterpart non-MINPs was measured and the results are shown in Fig. 2. As can be seen in Fig. 2, MINP obtained from run number 32 shows the highest drug loading in comparison with its counterpart non-MINP and hence it was selected for further characterization, in vitro and in vivo studies.

Figure 3 shows the FT-IR spectrum of unwashed and washed MINP-32 (Fig. 3a, b) and non-MINP-32 (Fig. 3c). As can be expected, $\mathrm{OH}$ stretching vibration of non-MINP and MINP-32 (washed) are strong at $3438.4 \mathrm{~cm}^{-1}$ and $3434.4 \mathrm{~cm}^{-1}$, respectively, whereas the $\mathrm{OH}$ vibration in MINP-32 (unwashed) has become weaker and transferred a little to the right $\left(3431.6 \mathrm{~cm}^{-1}\right)$.

TGA thermogram of unwashed and washed MINP-32 and non-MINP-32 are presented in Fig. 4. Non-MINP-32 started to decompose at a lower temperature (about $280^{\circ} \mathrm{C}$, Fig. 4d) than unwashed and washed MINP-32 (about 331$332^{\circ} \mathrm{C}$, Fig. $4 \mathrm{~b}, \mathrm{c}$ ). Olanzapine began to decompose at a temperature far below the temperature of polymer (about $220^{\circ} \mathrm{C}$, Fig. 4a).

Morphology of nano-particles obtained by SEM is illustrated in Fig. 5. The size of non-MINP-32 (Fig. 5a) is significantly bigger than washed and unwashed MINP-32 (279 nm versus $117-122 \mathrm{~nm}$, Fig. 5b, c) although there is no considerable difference in shape among them and all of them are almost spherical. These are almost in a good match with data obtained from size measurements obtained from photon correlation spectroscopy. The size for non-MINP-32 was measured $263 \pm 5 \mathrm{~nm}$ and for washed and unwashed MINPs 123 $\pm 3 \mathrm{~nm}$ and $119 \pm 4 \mathrm{~nm}$, respectively, with a poly-dispersity index of 0.2 to 0.43 . No significant difference was observed among Zeta potential of non-MINP-32, washed and unwashed MINP-32, and all was measured $6.9 \pm 0.4 \mathrm{mV}, 7.1 \pm 0.2 \mathrm{mV}$, and $6.8 \pm 0.4 \mathrm{mV}$, respectively.

According to the IUPAC classification (23), nitrogen adsorption-desorption isotherm of MINP-32 belongs to type IV isotherm which is an indication of a porous material 


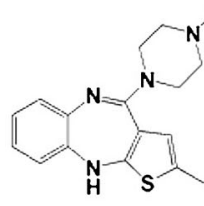<smiles>C=CC(=O)O</smiles>

Olanzapine

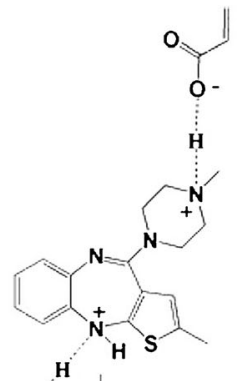

H o-
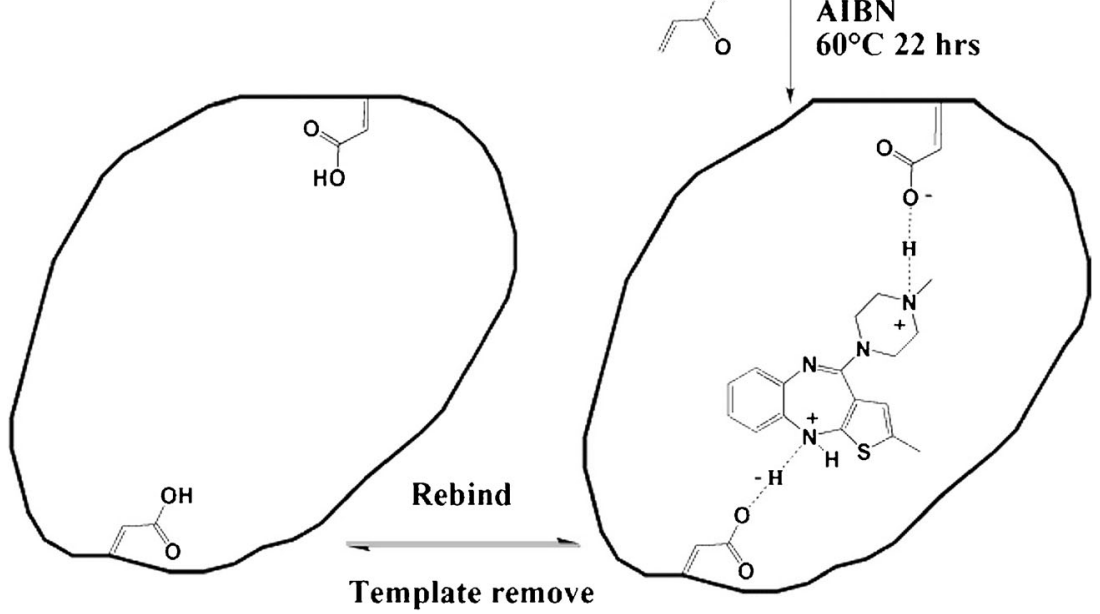

Fig. 1. Schematic illustration of MINPs synthesis

containing micropores $(\leq 2 \mathrm{~nm})$ and mesopores (2 to $50 \mathrm{~nm})$. During adsorption phase, at lower pressure region, micropores filling occur until they are filled by a nitrogen monolayer completely. Then the mesopores continue to be filled by capillary condensation as the pressure increases. During desorption phase, as pressure is lowered, the mesopores are discharged by capillary evaporation. The capillary condensation and capillary evaporation do not take place at the same pressures, hence a hysteresis loop is created. MINP-32 shows a large specific surface area which is confirmed by type IV isotherm with a hysteresis loop at $P / P O>0.3$.

\section{Optimization of Loading}

To find optimum conditions for loading of template in its maximum possible amount, three independent factors in two levels were studied. Seventeen runs were performed to study response surface according to Box-Behnken methodology as

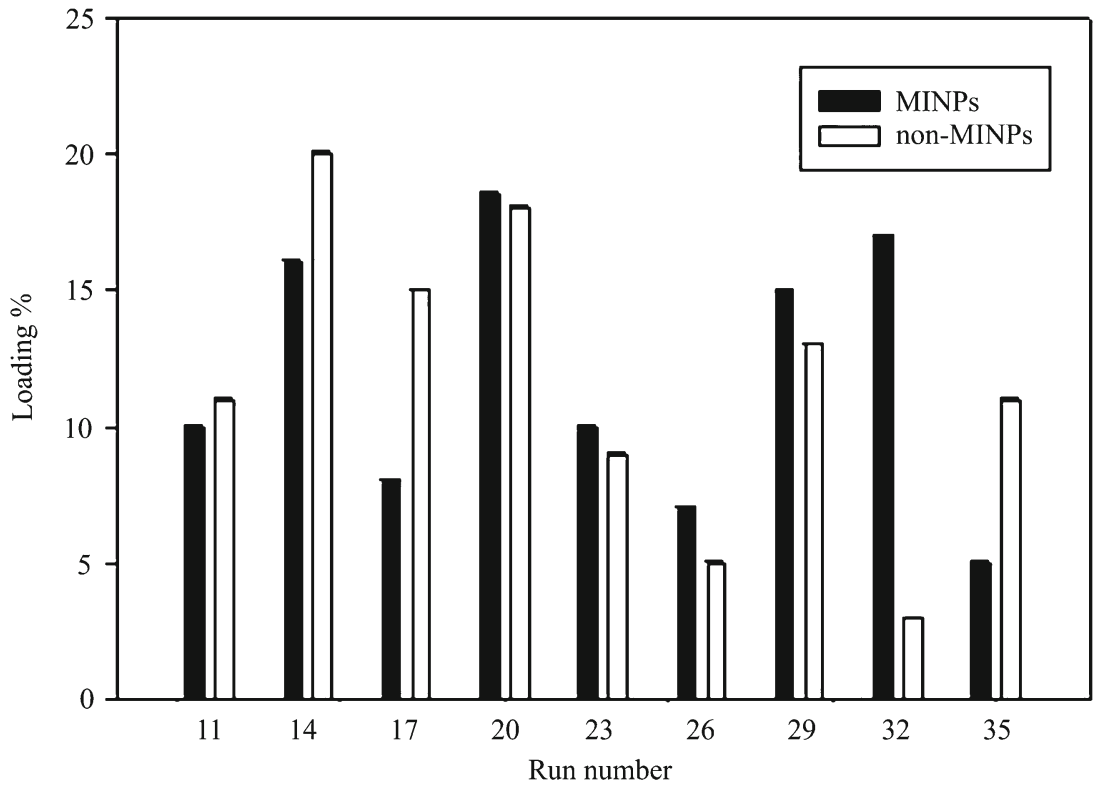

Fig. 2. Loading efficacy measurement of 9 MINPs and their counterpart non-MINPs (mean of 3 experiment \pm SD) 


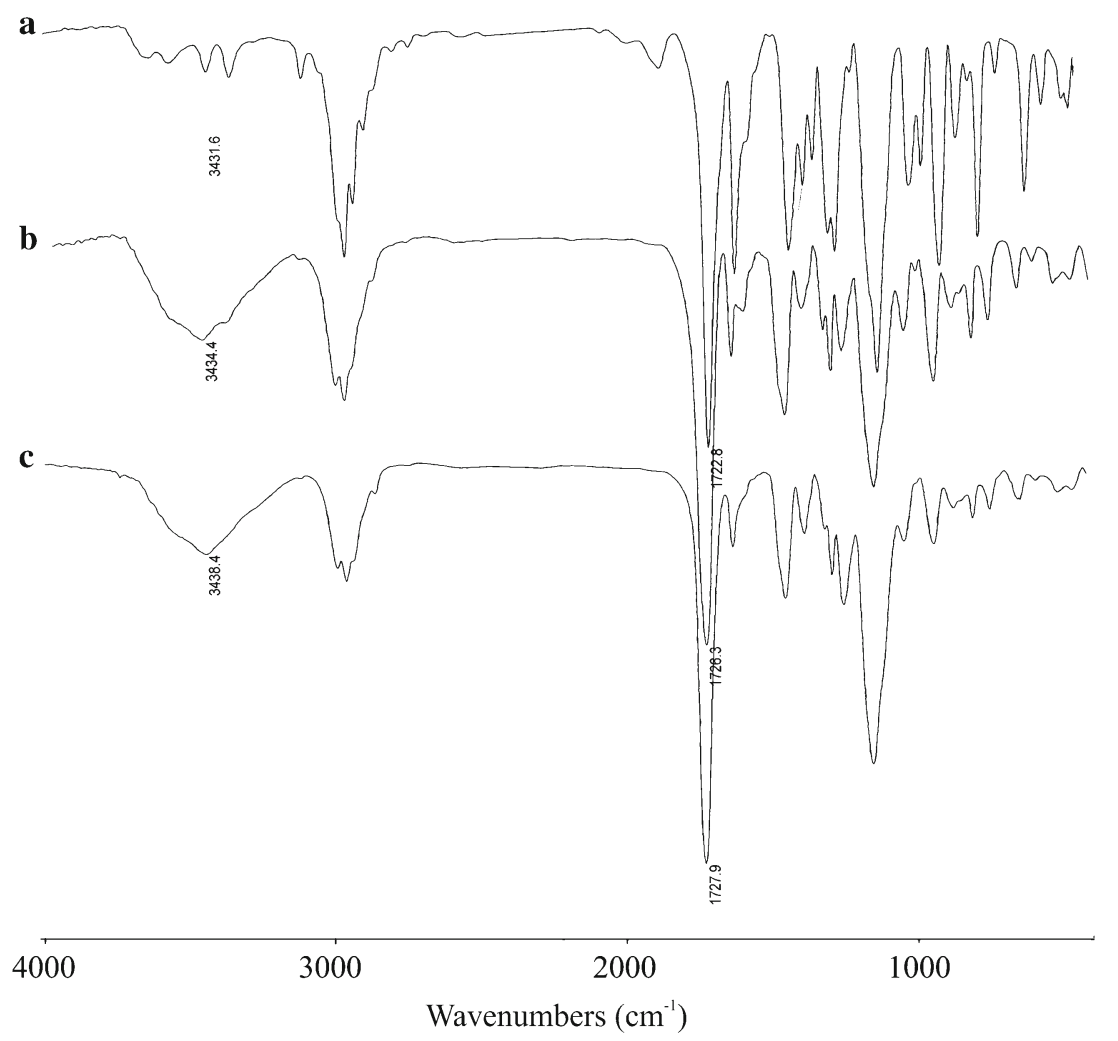

Fig. 3. FT-IR spectrums of unwashed MINP-32 (a), washed MINP-32 (b), and non-MINP-32 (c)

illustrated in Table II. Analysis of the data shows that the model is best fitted in quadratic model with a model $F$ value of 26.6 which is significant. There is only a $0.01 \%$ chance that a large model $F$ value like this could occur due to noise. Values of probability $>F$ less than 0.05 were assumed significant for model terms and "not significant" terms were discarded. Among the model terms, $\mathrm{pH}$ has the highest effect on template loading with the highest $F$ value. The "Lack of Fit $F$ value" of 3.63 implies the lack of fit is not significant relative to the pure error. There is an $11.54 \%$ chance that a large lack of fit $F$ value like this could occur due to noise. The value of 0.923612 for regression coefficient ( $R$-Squared) shows that regression unable to explain only $7.6 \%$ of the total variability due to human or experimental errors. The predicted $R$ Squared of 0.7797 is in reasonable agreement with the

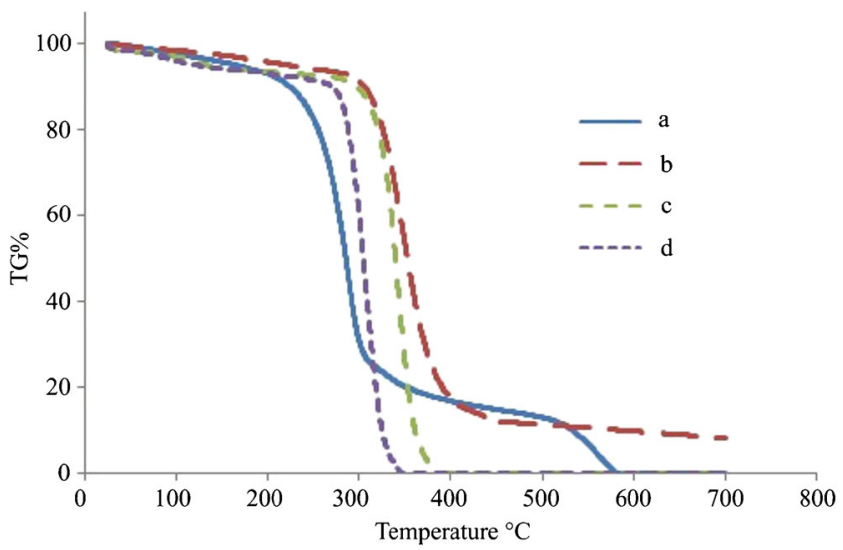

Fig. 4. TGA of olanzapine (a), unwashed MINP-32 (b), washed MINP-32 (c), and non-MINP-32 (d) adjusted $R$-Squared of 0.8889 . Adequate precision measures the signal to noise ratio. A ratio greater than 4 is desirable. Our ratio of 18.034 indicates an adequate signal. This model can be used to navigate the design space.

The effects of different factors on loading of template are illustrated in Fig. 6. As can be observed, $\mathrm{pH}$ has the maximum effect on loading, keeping time in its center value of $75 \mathrm{~min}$. In lower $\mathrm{pH}$, increasing the amount of absorbent does not show a significant increase in template loading. In contrast, in higher $\mathrm{pH}$, by increasing the amount of absorbent the loading has also increased (Fig. 6b). In a fixed $\mathrm{pH}$ value at center point $(\mathrm{pH}=6.0)$ by increasing the amounts of template and time, the loading has increased steadily (Fig. 6d). The effects of the template amount and time was not considerable at $\mathrm{pH}=1.0$, whereas at $\mathrm{pH}=11.0$, the effect of factors was considerable (data is not shown). In a fixed amount of template (center point), the effect of $\mathrm{pH}$ was more significant than time. The prominent effect of $\mathrm{pH}$ could be due to the physicochemical properties of template. Olanazapine is a weak base with a $\mathrm{pK}_{\mathrm{a}}$ of 7.4 and is more soluble in acidic conditions; therefore, it is more ionized and cannot establish an effective interaction with polymer matrix. In contrast, in alkaline conditions the template molecule is less ionized and can interact with polymer matrix, probably through hydrogen binding as it is proved by FT-IR.

Based on data analysis the model equation can be written as follows:

$$
\begin{aligned}
Y= & 0.040556 X_{i}-4.14528 X_{i i}+0.220833 X_{i i i}+0.583056 X_{i i}^{2} \\
& +0.051111 X_{i} X_{i i}-3.66694
\end{aligned}
$$



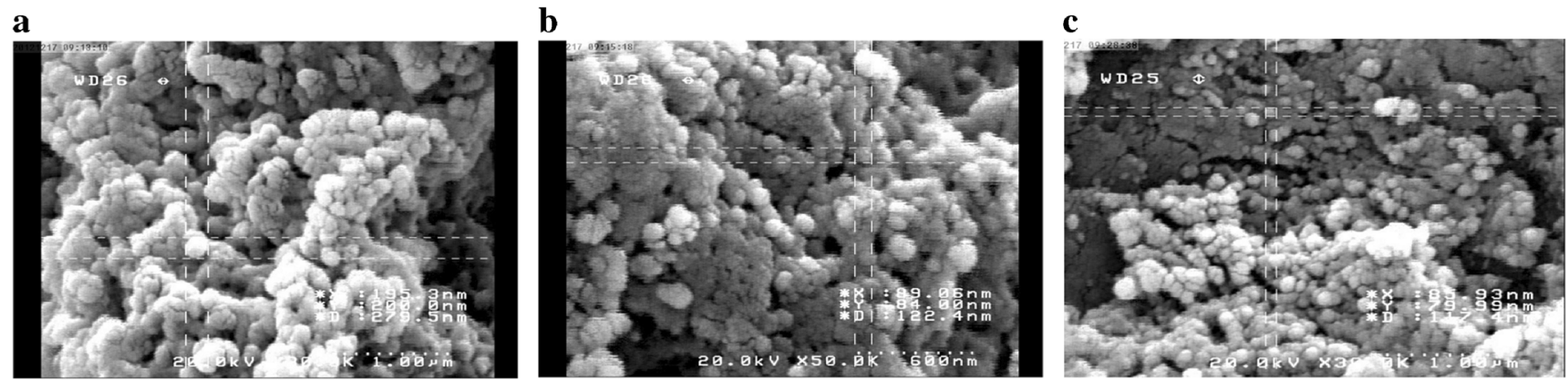

Fig. 5. SEM of non-MINP-32 (a, $\times 18,000)$, unwashed MINP-32 $(\mathbf{b}, \times 33,000)$, and washed template $(\mathbf{c}, \times 21,000)$

in which $Y$ is loading, and $X_{i}, X_{i i}$, and $X_{i i i}$ are absorbent amount, $\mathrm{pH}$, and time, respectively. In order to examine the validity of the model, three confirmation runs were performed according to the first suggested model solution for a loading of $85 \%$ within a range of $80-90 \%$ as lower and upper limits. Accordingly, $79 \mathrm{mg}$ was used at $\mathrm{pH}=10.90$ with a mixture time of $79 \mathrm{~min}$.

MINP-32 could have significantly distinguished between olanzapine and its analog clozapine in comparison with nonMINP-32. MINP-32 has absorbed about $85 \%$ of olanzapine and only $48 \%$ of clozapine, which imply almost a good selectivity of the polymer. In non-MINP-32, on the other hand, the ability of polymer to absorb olanzapine has decreased dramatically to $57 \%$ while for clozapine only to $42 \%$.

\section{In Vitro Study}

Figure 7 presents the template release profile from MINP-32 and non MINP-32. Both drug loaded MINP-32 and non MINP-32 show a burst release of 32.5 and $59 \%$ in about $24 \mathrm{~h}$, respectively. It could be due to the drug release from particles surface. The non MINP-32 releases almost all of its load (94\%) and reaches to a plateau after $120 \mathrm{~h}$ while MINP32 releases its load more slowly and reaches to its maximum release $(95 \%)$ after $288 \mathrm{~h}$.

Based on model fitting analysis for drug release from MINP-32, the release is best fitted in Korsmeyer and Peppas mathematical model with the highest regression coefficient $R_{\text {sqr }}$ in comparison with other models (0.9837 versus 0.9786 a

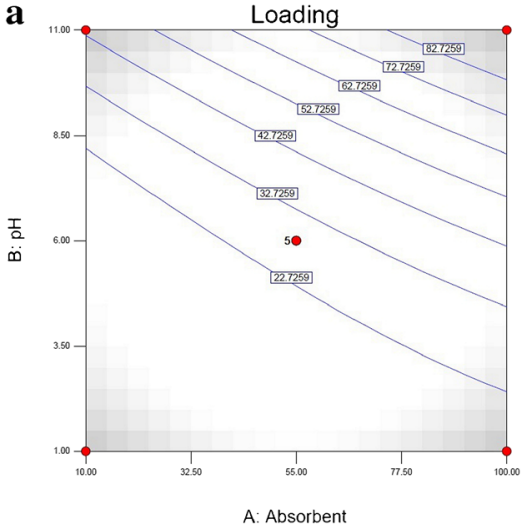

b

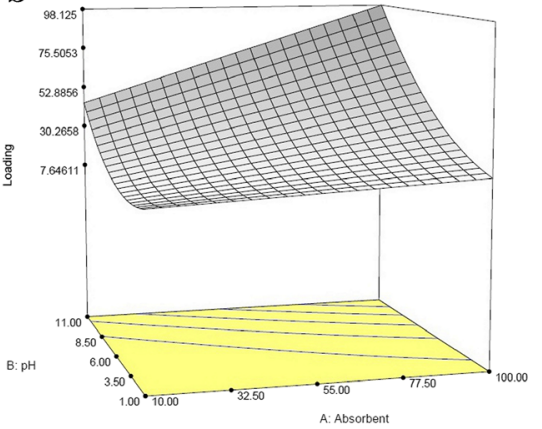

c

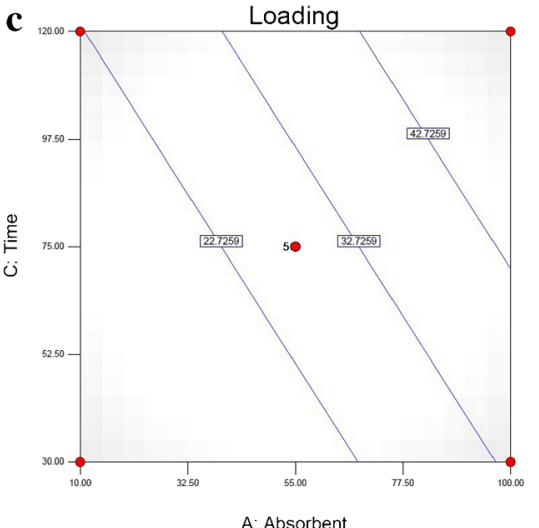

d

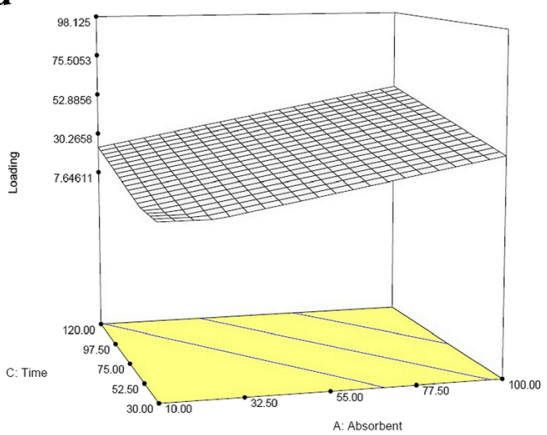

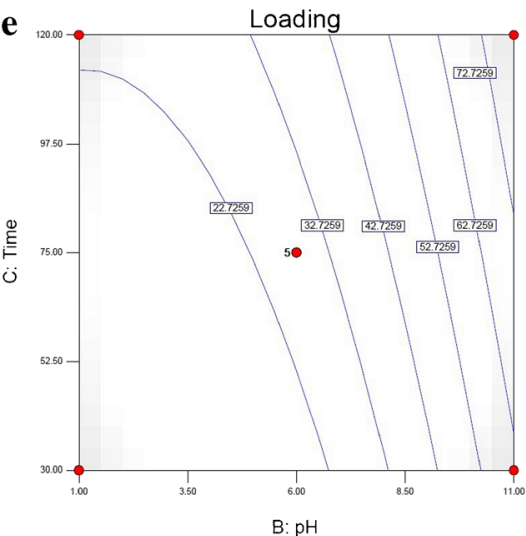

f

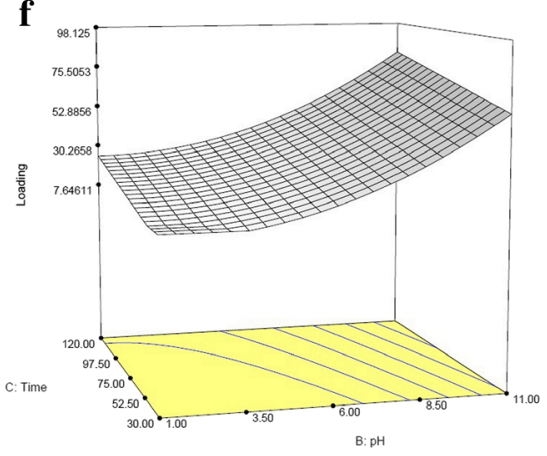

Fig. 6. Counter and three dimensional response surface plot of the effect of absorbent and $\mathrm{pH}(\mathbf{a}, \mathbf{b})$, absorbent and time (c, d), and $\mathrm{pH}$ and time $(\mathbf{e}, \mathbf{f})$ on loading of template 


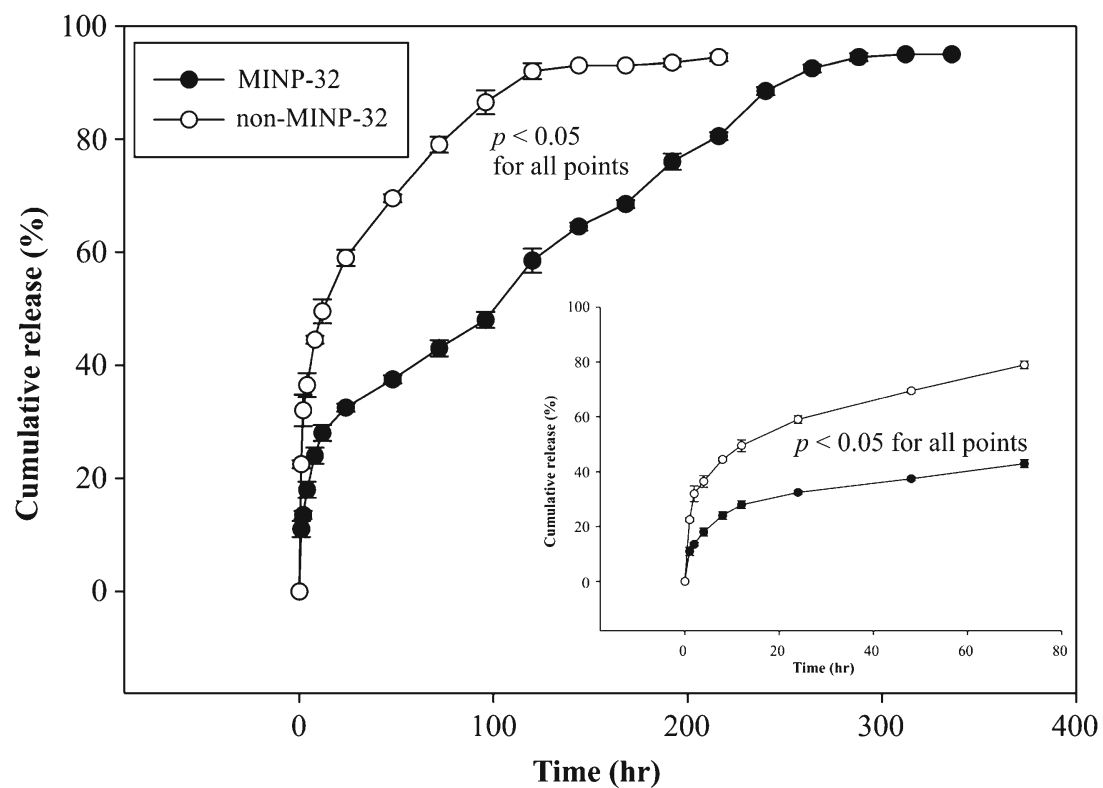

Fig. 7. Release profile of olanzapine from MINP-32 and non-MINP-32 (mean of 3 experiment \pm SD) (Student's $t$ test; $p<0.05$ is significant)

for Higuchi model and 0.9681 for Baker and Lonsdale) and adjusted regression coefficient (Adj $R_{\mathrm{sqr}}$ ) is in comply with $R_{\text {sqr. }}$

The amount of olanzapine in MINP-32 reduced not more than about $5 \%$ within one month kept at harsh condition of 50 $\pm 2{ }^{\circ} \mathrm{C} / 90 \pm 5 \mathrm{RH}$ compared to its initial amount before starting the study. An increase of less than 5\% was measured in total impurities with observing no additional impurities after elapsing the stability condition.

\section{In Vivo Study}

Figure 8 demonstrates the plasma concentration of MINP-32 after IM administration. After $24 \mathrm{~h}$, the serum concentration in the test group was measured $45-54 \mu \mathrm{g} / \mathrm{ml}$ in comparison to $90-100 \mu \mathrm{g} / \mathrm{ml}$ in control. These values after $72 \mathrm{~h}$ were $28-36$ and $31-45 \mu \mathrm{g} / \mathrm{ml}$, respectively. After $144 \mathrm{~h}$, the plasma level in the test group decreased to $20-28 \mu \mathrm{g} / \mathrm{ml}$ while in control group it decreased to $5-10 \mu \mathrm{g} / \mathrm{ml}$. After $216 \mathrm{~h}$ almost no drug was detectable in both test and control groups and therefore the experiments were terminated.

\section{DISCUSSION}

In FT-IR of MINP-32 (unwashed), $\mathrm{OH}$ vibration has become weaker and transferred a little to the right which could be due to hydrogen binding interaction between template and polymer matrix. The same phenomenon has observed and reported by others in the same polymeric system with the same composition but with different template (24). This is also confirmed by the TGA thermogram obtained from unwashed and washed MINP-32 and non-MINP-32. Polymeric matrix in MINP-32 has a more organized structure than nonMINP-32 and it is more robust than its non-MINP counterpart; therefore, it decomposes in higher temperatures than non-MINP.

As a result of interaction between template and polymer matrix it is expected that the size of MINP-32 particles would be smaller and that their shape would be more spherical than the non-MINP-32 particles. It is confirmed in size obtained from SEM as illustrated in "RESULTS" section. This could be due to synthesis method. To obtain homogenous and uniform nano-particles the reaction mixture was almost stirred vigorously throughout the whole reaction course in all runs. Therefore, there was no opportunity to have the primary particle cores formed in the beginning of the polymerization grown up and deviate from the spherical shape. In preliminary studies to set the polymerization conditions particles in micro scale with irregular shape were observed (data is not shown). In spite of olanzapine and polymer interaction, the stability of the template has not been affected by this interaction as can be seen from stability study. It confirms that there is no incompatibility between the template and polymer.

The MINP-32 has a very porous structure based on data obtained from nitrogen adsorption-desorption isotherm. With respect to the specific pore volume of $0.2315 \mathrm{~cm} 3 / \mathrm{g}$ and average pore diameter of $3.1004 \mathrm{~nm}$ on the one hand and specific

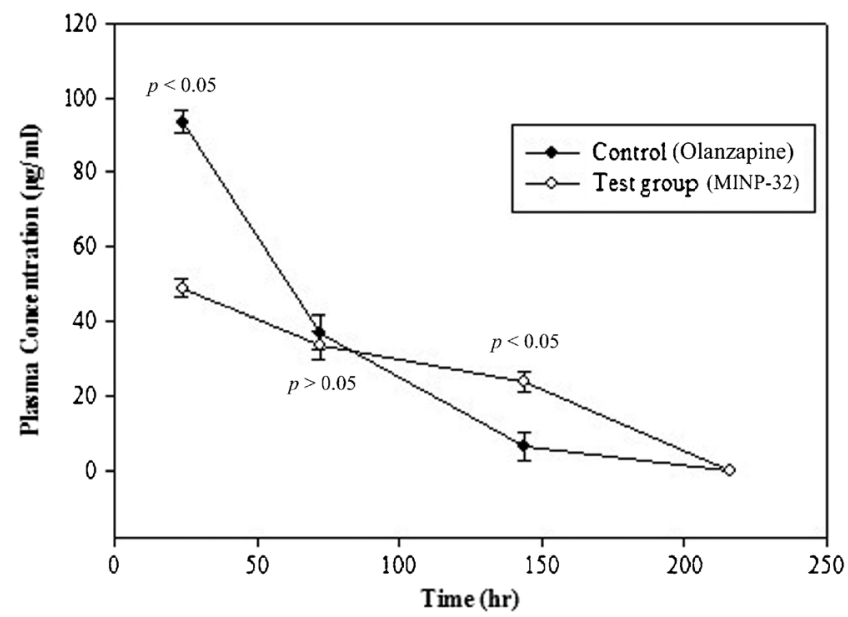

Fig. 8. Plasma concentration of olanzapine obtained from MINP-32 in comparison the olanzapine alone (mean of 3 experiment \pm SD) (Student's $t$ test; $p<0.05$ is significant) 
surface area of $298.64 \mathrm{~m} 2 / \mathrm{g}$ on the other hand, a very porous structure is expected for MINP-32. Adsorption capacity of $150.4 \mathrm{mg} / \mathrm{g}$ confirms this expectation. The results are in good compliance with research works in this field $(25,26)$.

The quadratic model used to optimize drug loading has a good power of prediction as examined for its validity. The amount of drug loading shows a maximum error of $3.55 \%$ which is acceptable. Therefore, it could be concluded that the quadratic model has a reasonable power of prediction within the experimental design range. The MINP-32 also showed a reasonable power of selectivity for its template in comparison with its analog.

The release profile of MINP-32 shows that it could have successfully kept the memory of the template and, hence, controlled the drug release due to effective weak interactions (e.g., electro-static and hydrogen bound) between polymer functional groups and template (27). It is in good compliance with other research works reported by researchers who were interested to control the release of templates of their choice using the same polymeric system with the same composition (28-30). The mechanism of dug release is best fitted on Korsmeyer-Peppas model with exponent $(n)$ value below 0.5 which reveals a quasi-Fickian transport from the NPs (31).

These results of animal study indicate that MINP-32 has the ability to control the release of its template. In test group, concentration of olanzapine decreases in a controlled manner within a longer time than control group. This data together with the release profile shows that MINP-32 has a desirable interaction with template to hold it for a long time and can be used as a controlled release drug delivery system for olanzapine.

\section{CONCLUSION}

In this research work MIMPs were synthesized for olanzapine as template. Based on our knowledge no research has yet been done in this field on olanzapine, and it is the first time a molecular imprinted system is made for this template. The results show that the developed MINP has a desirable memory of the template and has the ability to control the release. The selected MINP can also maintain its properties of keeping the memory of the template and controlling its release in biological environment.

Therefore, the MINPs of this research work could be a promising delivery system for olanzapine. Due to long-term in vitro release and in vivo plasma concentration profile of this delivery system it is expected that the system could be applied as intramuscular injection. More studies are under progress to assess toxicity properties of the system and its performance in long term stability studies, as well as its ability to be applied as subcutaneous injection or topical preparations such as dermal patches.

\section{ACKNOWLEDGMENTS}

We would like to acknowledge Dr. Abdollah Asadi [Department of Chemical Engineering, Amirkabir University of Technology (Tehran Polytechnic), Tehran, Iran] for his useful advices in synthesis of the MINPs and Niloofar Babanejad (Chemistry Department, Faculty of Sciences, IKIU, Qazvin, Iran) for her assistance in taking TGA thermograms and in vitro study. This research work was supported financially
Tehran University of Medical Sciences (registration No. 8903-33-11330).

\section{COMPLIANCE WITH ETHICAL STANDARDS}

Conflict of Interest

The authors declare that they have no

competing interests.

\section{REFERENCES}

1. Kim S, Kim JH, Jeon O, Kwon IC, Park K. Engineered polymers for advanced drug delivery. Eur J Pharm Biopharm Off $\mathbf{J}$ Arbeitsgemeinschaft Pharma Verfahrenstechnik eV. 2009;71(3):420-30.

2. Dinarvand R, Dorkoosh F, Hamidi M, Moghadam SH. Polymeric delivery systems for biopharmaceuticals. Biotechnol Genet Eng Rev. 2004;21(1):147-82.

3. Davis SS, Illum L, Stolnik S. Polymers in drug delivery. Curr Opin Colloid Interface Sci. 1996;1(5):660-6. English.

4. Pillai O, Panchagnula R. Polymers in drug delivery. Curr Opin Chem Biol. 2001;5:447-51. English.

5. Sellergren B, Allender CJ. Molecularly imprinted polymers: a bridge to advanced drug delivery. Adv Drug Deliv Rev. 2005;57(12):1733-41.

6. Takeuchi T, Haginaka J. Separation and sensing based on molecular recognition using molecularly imprinted polymers. J Chromatogr B. 1999;728:1-20. English.

7. Xu X, Zhu L, Chen L. Separation and screening of compounds of biological origin using molecularly imprinted polymers. J Chromatogr B Anal Technol Biomed Life Sci. 2004:804(1):61-9.

8. Ansell RJ, Kuah JK, Wang D, Jackson CE, Bartle KD, Clifford AA. Imprinted polymers for chiral resolution of (+/-)-ephedrine, 4: packed column supercritical fluid chromatography using molecularly imprinted chiral stationary phases. J Chromatogr A. 2012;1264:117-23

9. Shi X, Wu A, Zheng S, Li R, Zhang D. Molecularly imprinted polymer microspheres for solid-phase extraction of chloramphenicol residues in foods. J Chromatogr B Anal Technol Biomed Life Sci. 2007:850(1-2):24-30.

10. Rathbone DL. Molecularly imprinted polymers in the drug discovery process. Adv Drug Deliv Rev. 2005;57(12):1854-74.

11. Sergeyeva TA, Slinchenko OA, Gorbach LA, Matyushov VF, Brovko OO, Piletsky SA, et al. Catalytic molecularly imprinted polymer membranes: development of the biomimetic sensor for phenols detection. Anal Chim Acta. 2010;659(1-2):274-9.

12. Cunliffe D, Kirby A, Alexander C. Molecularly imprinted drug delivery systems. Adv Drug Deliv Rev. 2005;57(12):1836-53.

13. Alvarez-Lorenzo C, Concheiro A. Smart drug delivery systems: from fundamentals to the clinic. Chem Commun (Camb). 2014:50(58):7743-65.

14. Mayes AG, Whitcombe MJ. Synthetic strategies for the generation of molecularly imprinted organic polymers. Adv Drug Deliv Rev. 2005;57(12):1742-78.

15. Vasapollo G, Sole RD, Mergola L, Lazzoi MR, Scardino A, Scorrano S, et al. Molecularly imprinted polymers: present and future prospective. Int J Mol Sci. 2011;12(9):5908-45.

16. Os JV, Kapur S. Schizophrenia. Lancet. 2009;374:635-45.

17. Picchioni MM, Murray RM. Schizophrenia. BMJ. 2007;335(7610):91-5.

18. Keith S. Advances in psychotropic formulations. Prog NeuroPsychopharmacol Biol Psychiatry. 2006;30(6):996-1008.

19. Kumar S, Kumar A, Gupta V, Malodia K, Rakha P. Oral extended release drug delivery system a promising approach. Asian J Pharm Technol. 2012;2(2):38-43. English.

20. Dash S, Murthy PN, Nath L, Chowdhury P. Kinetic modeling on drug release from controlled drug delivery systems. Acta Pol Pharm Drug Res. 2010;67(3):217-23. English.

21. Higuchi T, Connors KA. Phase-solubility techniques. Adv Anal Chem Instrum. 1965;4:117-22. English. 
22. Korsmyer RW, Gumy R, Doelker E, Buri P, Peppas NA. Mechanisms of solute release from porous hydrophilic polymers. Int $\mathrm{J}$ Pharm. 1983;15:25-35. English.

23. Sing KSW, Everett DH, Haul RAW, Moscou L, Pierotti RA, Rouquerol J, et al. Reporting physisorption data for gas-solid systems with specific reference to the determination of surface area and porosity. Pure Appl Chem. 1985;57(4):603-19. English.

24. Seifi M, Hassanpour Moghadam M, Hadizadeh F, Ali-Asgari S, Aboli J, Mohajeri SA. Preparation and study of tramadol imprinted micro-and nanoparticles by precipitation polymerization: microwave irradiation and conventional heating method. Int J Pharm. 2014;471(1-2):37-44.

25. Wei S, Molinelli A, Mizaikoff B. Molecularly imprinted micro and nanospheres for the selective recognition of 17beta-estradiol. Biosens Bioelectron. 2006;21(10):1943-51.

26. Chen J, Bai LY, Liu KF, Liu RQ, Zhang YP. Atrazine molecular imprinted polymers: comparative analysis by far-infrared and ultraviolet induced polymerization. Int J Mol Sci. 2014;15(1):574-87.

27. Lulinski P. Molecularly imprinted polymers as the future drug delivery devices.pdf. Acta Pol Pharm Drug Res. 2013;70(4):6019. English.

28. Puoci F, Iemma F, Cirillo G, Picci N, Matricardi P, Alhaique F. Molecularly imprinted polymers for 5-fluorouracil release in biological fluids. Molecules. 2007;12:805-14. English.

29. Puoci F, Cirillo G, Curcio M, Iemma F, Parisi OI, Castiglione M, et al. Molecularly imprinted polymers for alpha-tocopherol delivery. Drug Deliv. 2008;15(4):253-8.

30. Cirillo G, Iemma F, Puoci F, Parisi OI, Curcio M, Spizzirri UG, et al. Imprinted hydrophilic nanospheres as drug delivery systems for 5fluorouracil sustained release. J Drug Target. 2009;17(1):72-7.

31. El-Leithy ES, Shaker DS, Ghorab MK, Abdel-Rashid RS. Optimization and characterization of diclofenac sodium microspheres prepared by a modified coacervation method. Drug Discov Ther. 2010;4(3):208-16. English. 